\title{
Correction: Recommendations From the Twitter Hashtag \#DoctorsAreDickheads: Qualitative Analysis
}

Anjana Estelle Sharma ${ }^{1,2}$, MAS, MD; Ziva Mann ${ }^{3}$, MA; Roy Cherian ${ }^{2,4}$, MHS; Jan Bing Del Rosario ${ }^{1,5}$, BS; Janine Yang $^{1,6}$, BS; Urmimala Sarkar ${ }^{2}$, MD, MPH

${ }^{1}$ Department of Family \& Community Medicine, University of California San Francisco, San Francisco, CA, United States

${ }^{2}$ Center for Vulnerable Populations, University of California San Francisco, San Francisco, CA, United States

${ }^{3}$ Ziva Mann Consulting, Newton, MA, United States

${ }^{4}$ Department of Culture and Theory, School of Humanities, University of California, Irvine, Irvine, CA, United States

${ }^{5}$ Berkeley School of Public Health, University of California Berkeley, Berkeley, CA, United States

${ }^{6}$ Drexel University College of Medicine, Philadelphia, PA, United States

\section{Corresponding Author:}

Anjana Estelle Sharma, MAS, MD

Department of Family \& Community Medicine

University of California San Francisco

995 Potrero Ave

Ward 83

San Francisco, CA, 94110

United States

Phone: 16179454776

Email: anjana.sharma@ucsf.edu

\section{Related Article:}

Correction of: https://www.jmir.org/2020/10/e17595/

(J Med Internet Res 2020;22(11):e25511) doi: 10.2196/25511

In "Recommendations From the Twitter Hashtag \#DoctorsAreDickheads: Qualitative Analysis (J Med Internet Res 2020;22(10):e17595), the authors noted three errors.

In the originally published manuscript, the fourth sentence of the second paragraph of the Introduction section read:

The term originated from a professional YouTube video maker, who posted a video on Twitter explaining that she had been diagnosed with Ehlers-Danlos syndrome and postural orthostatic hypotension syndrome (POTS).

This has been changed to:

The term originated from a professional YouTube video maker, who posted a video on Twitter explaining that she had been diagnosed with Ehlers-Danlos syndrome and postural orthostatic tachycardia syndrome (POTS).
In Table 2, footnote ${ }^{\text {a }}$ originally read:

POTS: postural orthostatic hypotension syndrome.

This has been changed to:

POTS: postural orthostatic tachycardia syndrome.

The Abbreviations section originally included the following:

POTS: postural orthostatic hypotension syndrome.

This has been changed to:

POTS: postural orthostatic tachycardia syndrome.

These corrections will appear in the online version of the paper on the JMIR Publications website on November 9, 2020, together with the publication of this correction notice. Because this was made after submission to PubMed, PubMed Central, and other full-text repositories, the corrected article has also been resubmitted to those repositories. 


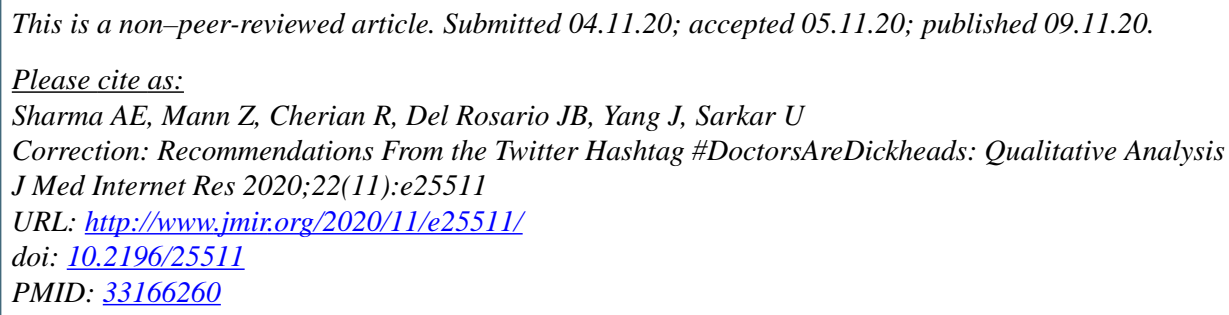

(C)Anjana Estelle Sharma, Ziva Mann, Roy Cherian, Jan Bing Del Rosario, Janine Yang, Urmimala Sarkar. Originally published in the Journal of Medical Internet Research (http://www.jmir.org), 09.11.2020. This is an open-access article distributed under the terms of the Creative Commons Attribution License (https://creativecommons.org/licenses/by/4.0/), which permits unrestricted use, distribution, and reproduction in any medium, provided the original work, first published in the Journal of Medical Internet Research, is properly cited. The complete bibliographic information, a link to the original publication on http://www.jmir.org/, as well as this copyright and license information must be included. 